\title{
An Assessment on the future development of high-entropy alloys: summary from a recent workshop
}

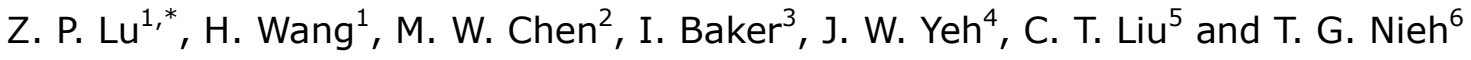 \\ ${ }^{1}$ State Key Laboratory for Advanced Metals and Materials, University of Science and Technology \\ Beijing, Beijing, 100083, China, 100083 \\ ${ }^{2}$ Advanced Institute for Materials Research, Tohoku University, Sendai 980-8577, Japan \\ ${ }^{3}$ Thayer School of Engineering, 14 Engineering Dr., Dartmouth College, Hanover, NH 03755, USA \\ ${ }^{4}$ Department of Materials Science and Engineering, National TsingHua University, Taiwan \\ ${ }^{5}$ Center of Advanced Structural Materials, Department of Mechanical and Biomedical Engineering, \\ College of Science and Engineering, City University of Hong Kong, Kowloon, Hong Kong, China \\ ${ }^{6}$ Department of Materials Science and Engineering, the University of Tennessee, Knoxville, TN 37996, \\ USA
}

\begin{abstract}
There is increasing interest in both relating the mechanical behavior of high-entropy alloys to their microstructural evolution and in their development for various applications. A special two-day international workshop on the above topic was held in Guiyang, China, in December 2014. The workshop gathered scientists and engineers to exchange information on recent progress in high-entropy alloys, to discuss the scientific issues and challenges to foster international collaborations, and to identify future directions. In this paper, a summary of this workshop is presented, including aspects of definition/terminology, phase formation, microstructure and phase stability, strengthening mechanisms, and high-temperature properties. Future research directions are also outlined.
\end{abstract}

*Email: luzp@ustb.edu.cn 


\section{Synopsis}

The continued development of high-performance materials is required for increased energy efficiency and sustainability. Traditionally, metallic alloys have been widely applied as structural materials. The conventional alloy design strategy usually is to start with one element as the principal constituent and adding other minor elements for the further optimization of properties and performances. After extensive efforts on these traditional alloys, the development of traditional alloys is approaching its limits. Recently, a new alloy design concept was proposed: the idea is to simultaneously alloy several principal elements (usually $\geq 5$ ) to obtain high mixing entropy. As a result, many novel concentrated multicomponent alloys are in the process of being developed [1-6]. In a timely response to this rapid development, a two-day international workshop was held in Guiyang, PRC in December 2014 to exchange information on recent progress, foster international collaborations, and identify future research directions. The main theme of the workshop was the relationship between microstructures and mechanical properties. In this paper, a summary of this special workshop is presented, which includes;

1) definition/terminology,

2) main characteristics resulting from multiple primary constituents,

3) atomic size effects on phase formation,

4) microstructure and phase stability,

5) strengthening mechanisms,

6) high-temperature mechanical properties, and

7) future directions for research 


\section{Definitions/terminology}

Although "high-entropy alloys (HEAs)" have now become a new class of materials, the name has not been clearly defined since its inception. This often causes confusion in the scientific community.

A definition was first proposed in 2004 based solely on the compositional requirements [2]. HEAs were defined as those alloys having at least five major metallic elements each having an atomic percentage between $5 \%$ and $35 \%$. This definition implicitly includes alloys with minor elemental additions with atomic percentages even smaller than $5 \%$. The expanded definition could be expressed as

$$
n_{\text {major }} \geqslant 5, \quad 5 \% \leqslant X_{i} \leqslant 35 \% \text { and } n_{\text {minor }} \geqslant 0, X_{j} \leqslant 5 \%,
$$

where $n_{\text {major }}$ and $n_{\text {minor }}$ are the number of major and minor elements, respectively. $X_{i}$ and $X_{j}$ are atomic percentages of the $i$ major element and $j$ minor element, respectively.

Under this definition, a HEA system could have three types of compositions. For example, the Hf-Nb-Ta-Ti-Zr HEA system has one equimolar composition, numerous non-equimolar compositions and numerous minor elemental additions. The underlying reason for this definition is to utilize high mixing entropy to enhance the formation of solid solution phases.

A second definition was based on the entropy concept, which also appeared in both the first and several follow-on papers [4, 8]. It defines HEAs as having a configurational entropy larger than $1.5 \mathrm{R}$ in their random solution state, where $\mathrm{R}$ is the gas constant. This definition could be expressed as

$$
\Delta S_{\text {conf }} \geq 1.5 R
$$

The calculation of configurational entropy per mole for a solid solution formed from $n$ 
elements is approximately:

$$
\Delta \mathrm{S}_{\mathrm{conf}}=-\mathrm{R} \sum_{\mathrm{i}=1}^{\mathrm{n}} x_{i} \ln x_{i}
$$

Where $x_{i}$ is mole fraction of the $i$ element in the random solution state. The underlying reason for this definition is that a configurational entropy (the largest contribution to the mixing entropy) larger than $1.5 \mathrm{R}$ would endow the material with a higher tendency to form a solid solution. From Richards' rule for metallic melting, the entropy change per mole, $\Delta \mathrm{S}_{\mathrm{f}}$, from solid to liquid is roughly $\mathrm{R}$. Based on the relation, $T_{\mathrm{m}} \Delta \mathrm{S}_{\mathrm{f}}=\Delta \mathrm{H}_{\mathrm{f}}$, at the melting point $T_{m}$, this implies that a free energy decrease of $R T_{m}$ is equal to the free energy increase of around one twelfth of the chemical bonding energy per mole during melting. Since the excess chemical bonding energy per mole (mixing enthalpy) is a fraction of chemical bonding energy per mole, it can be expected that a HEA having a configurational entropy of at least $1.5 \mathrm{R}$ in the random solution state should enhance the formation of disordered solid solution at elevated temperatures as opposed to the formation of ordered structures due to negative mixing enthalpy and segregation due to positive mixing enthalpy.

In some cases, the aforementioned two definitions appear to be similar but are not exactly the same. For example, the alloy $\mathrm{Fe}_{5.9} \mathrm{Co}_{29.4} \mathrm{Cr}_{29.4} \mathrm{Ni}_{29.4} \mathrm{Cu}_{5.9}$ (at. \%) could be regarded as a HEA based on the compositional definition, but not according to the entropy definition since its configurational entropy is only $1.414 \mathrm{R}$.

Both definitions are simply approximate guidelines. Alloys with a small deviation in composition from the above definitions might also be regarded as HEAs. A similar situation, in fact, also exists in other material disciplines. For example, in the field of grain boundary engineering, the division between a high-angle boundary and low-grain boundary is somewhat arbitrary, yet there is general agreement that the transition is at $15^{\circ}$. Another example is the definition of superplasticity, which is defined as having a tensile elongation over $300 \%$. The definition is actually phenomenological and the number was 
again somewhat arbitrarily chosen, but with an agreement in the scientific community. It is noted that, within the materials community, other terminologies have also been proposed to replace HEAs, such as concentrated multicomponent alloys, compositionally complex alloys and baseless alloys (or multi-base alloy). However, it is felt that the notation "high-entropy alloys" should be adopted, since it is now a recognizable name in the community. It represents a class of materials that comprise multiple principal elements, instead of a single base metal as in the traditional alloys. Also, at the present time, a clear, irrevocable definition may not be necessary in order to allow researchers have a greater flexibility to explore the field.

\section{Several major characteristics}

Four core effects that are present in HEAs, including high-entropy, sluggish diffusion, severe lattice distortion and the cocktail effect, were first proposed in 2006 because of their uniqueness in metallic alloy [7, 8]. First, the high entropy effect enhances the formation of solid solution phases especially at high temperatures. This effect is important in reducing the number of phases in HEAs. Otherwise, their microstructure would become complex and likely brittle. Second, lattice diffusion in HEAs is sluggish, even at high temperatures. The slow lattice diffusion in the FeCoNiCrMn HEA has been studied systematically [9] and is shown in Fig. 1; each of the five components in this HEA indeed has a relatively lower diffusion coefficient compared to that in conventional alloy matrices. Also, sluggish diffusion reduces phase transformation rates. Thirdly, HEAs usually possess severe lattice distortion $[6,10]$. In HEAs, the terms "solute" and "solvent" lose their conventional meanings. The differences in the atomic sizes and bonding among various constituents in this kind of 'super solid-solution' systems introduce severe lattice distortion. Such severe lattice distortion affects both the 
microstructure and properties, and in this kind of distorted lattices, the point defects, line defects, and planar defects would be different from those in conventional matrices with respect to their atomic configuration, defect energy, and dynamic behavior which includes the mobility of the defects, interactions between defects, and interactions between defects and the lattice. This effect will be further discussed in the following paragraph. The cocktail effect, or synergistic effect, is the overall effect resulted from mutual interactions among composing elements, which would bring excess quantities to the average values simply predicted by the mixture rule.

Among the four core effects, lattice distortion effect has both direct and indirect influences on many aspects of the microstructure and properties. In a crystalline structure with multiple elements such as in HEAs, the lattice distortion can be caused by the differences in atomic size, crystal structure, and chemical bonding. Lattice distortion causes an atom at its specific lattice site, in principle, deviate from its ideal location. In addition, the electron configuration around any atom at a lattice site would have no exact symmetry as compared with that in ideal crystal structure of pure component since each atom bonds with neighboring atoms of different elements in HEAs. Obviously, the site deviation and non-symmetrical electron distribution depend on the interatomic potentials with its neighboring atoms. Thus, the overall distortion of the lattice has these two parts: topological distortion and physicochemical distortion. In topological distortion, the position deviation from equilibrium site could induce strain energy, which could result in larger resistance to dislocation motion, and affect the diffraction of incident beams such as X-rays and neutrons causing diffuse scattering. In physicochemical distortion, the non-symmetrical electron distribution essentially does not change the bond strength of each pair but could affect the diffraction of incident beams and free-electron migration by scattering. In brief, all these are expected to produce direct or indirect effects on the microstructure and properties in a yet-to-determined manner. 


\section{Atomic size effects on phase formation (Z .J. Wang, C. T. Liu, and Yong Yang)}

Phase formation and the selection of constituent elements in HEAs are complex physical metallurgy problems and influenced by many factors such as atomic size differences, mixing enthalpy, configurational entropy, chemical bonding, valence electron concentration, and so on. In this workshop, the effects of atomic size were heavily discussed, due to the fact that it is one of the most important factors in determining the lattice distortion, sluggish diffusion and phase stability.

Over a half of century ago, the classical Hume-Rothery rules summarized the physical parameters for the formation of solid solutions [11], where the atomic size mismatch is the first piece of the puzzle. As stated, a binary solid solution is unstable if the atomic size factor $\left|r_{A}-r_{B}\right| / r_{B}$ exceeds $15 \%$, where $r_{A}$ and $r_{B}$ are the atomic radius of the solute and solvent atoms, respectively. This criterion has been theoretically treated by Eshel by through continuum elastic theory [12]. However, the atomic size factor of the Hume-Rothery rules mainly considers binary alloys with a single principal solvent element. For HEAs with multiple primary elements, however, a new, effective parameter must be adopted, most likely through a statistical approach. For simplicity, the standard deviation of atomic sizes defined as

$$
\delta=\sqrt{\sum_{i}^{n} c_{i}\left(1-r_{i} / \bar{r}\right)^{2}}, \bar{r}=\sum_{i}^{n} c_{i} r_{i},
$$

has been widely accepted to describe effect of the atomic-size difference on the structural instability [13], where $c_{i}$ is the atomic concentration and $r_{i}$ is the atomic radius of the $i^{\text {th }}$ atom. Although the $\delta$ parameter has been commonly used in HEAs $[6,13,14]$, proper understanding of the underlying mechanisms of the atomic size effect is still lacking [16-18], and an atomic-size parameter with a clear physical meaning has been 
absent for a long time.

Recently, a new parameter, $\gamma$, was proposed with consideration of the solubility of an element in HEAs containing multiple base metals [19]. According to the literature [20, 21], it is known that the atomic-size difference influences the topological instability of atomic packing. It was argued that the atoms with the maximum and minimum sizes play a decisive role in determining the packing stability in HEAs [19].The solid angles of atomic packing for the elements with the largest $\left(r_{L}\right)$ and smallest atomic sizes $\left(r_{S}\right)$ are chosen to quantitatively describe the atomic packing effects in the multicomponent alloys. Then, a normalized parameter of the packing state, defined as the ratio between the solid angles for the smallest and largest atoms,

$$
\gamma=\omega_{S} / \omega_{L}=\left(1-\sqrt{\frac{\left(r_{S}+\bar{r}\right)^{2}-\bar{r}^{2}}{\left(r_{S}+\bar{r}\right)^{2}}}\right) /\left(1-\sqrt{\frac{\left(r_{L}+\bar{r}\right)^{2}-\bar{r}^{2}}{\left(r_{L}+\bar{r}\right)^{2}}}\right)
$$

was chosen as an indicator to reveal the atomic packing misfit and topological instability in the HEAs.

More recently, two new parameters, $\alpha_{1}$ and $\alpha_{2}$, were also proposed based on a description of the lattice distortion and intrinsic strain energy [22]. The lattice distortion is defined by comparing a distorted lattice and its ideal counterpart lattice. In a random solution, the average radius $\bar{r}$ can be used to characterize a uniform ideal lattice. With the assumption that the ideal lattice represents the reference state, $\left|r_{i}-\bar{r}\right| / \bar{r}$ will be the dimensionless displacement of an atom from an ideal atom, and the total displacement will be

$$
\alpha_{1}=\sum_{i}^{n} \frac{c_{i}\left|r_{i}-\bar{r}\right|}{\bar{r}}
$$


By defining the $\left|r_{i}+r_{j}-2 \bar{r}\right| / 2 \bar{r}$ as the dimensionless displacement between an atomic pair and its counterpart pair, then the parameter $\alpha_{2}$ represents a local atomic distortion:

$$
\alpha_{2}=\sum_{j \geq i}^{n} \frac{c_{i} c_{j}\left|r_{i}+r_{j}-2 \bar{r}\right|}{2 \bar{r}}
$$

Generally, there are three main categories of phase regions in as-cast HEAs: solid solutions, solid solution plus intermetallic compounds, and metallic glasses. Figure 2 shows the $\gamma-\delta$ plot of representative as-cast HEAs reported recently [14], and it can be seen that the $\gamma$ parameter can clearly distinguish the alloys of solid solutions, solid solution plus intermetallics from metallic glasses using the cut-off value $\gamma=1.17$. For the conventional parameter $\delta$, there is an overlapping region of solid solutions and solid solutions plus intermetallics, i.e., $0.04<\delta<0.07$. In Fig. 2, both parameters, $\gamma$ and $\delta$, can well separate the metallic glasses from solid solutions. It is remarkable that all the newly developed high-entropy metallic glasses (MG-HEAs) have a distinct large $\gamma$. In these MG-HEAs [23-28], small substitutional atoms are involved, and hence the resultant high topological instability promoted the formation of metallic glasses. The data from all known alloys indicate that $\gamma$ is a better parameter to describe phase selection of HEAs and quite effective in extending the atomic size criterion of the Hume-Rothery rules from binary to HEAs with multiple bases.

Figure 3 shows the phase selection map against the lattice distortion parameters $\alpha_{1}$ and $\alpha_{2}$ in as-cast HEAs. The solid solutions generally exist at smaller $\alpha_{1}$ and $\alpha_{2}$, and both two parameters can well separate the region of solid solution phases from that of the solid-solution plus intermetallic compounds. Nevertheless, $\alpha_{2}$ is much better than 
$\alpha_{1}$ for distinguishing the region of metallic glasses from that of the solid solution plus intermetallics, as demonstrated in Fig. 3. The main reason is that the local lattice distortion is generally related to a group of adjacent atoms, and $\alpha_{2}$ is better in representing the lattice distortion among atomic pairs.

\section{Microstructure and phase stability}

The microstructures of HEAs span a wide range from single phase to multiphase: while adding additional elements increases the configurational entropy, sometimes, it can also cause a transition from single phase to multiphase depending on the specific element added and its amount. This has been demonstrated in the classic CoCrCuFeNi system. Cast equiatomic $\mathrm{CoCrCuFeNi}$ has a fcc-structure, but for $\mathrm{CoCrCuFeNiAl}$, where all the elements apart from the $\mathrm{Al}$ are in equiatomic amounts and $x$ varies from 0 to 3 , increasing the amount of $\mathrm{Al}$ causes a transition from single phase fcc (at $x \geqslant 0.5$ ) to mixed fcc $+\mathrm{bcc}$, and eventually to single-phase bcc (at $x=2.8$ ). Al is a well-known bcc stabilizer, and its addition to $\mathrm{CoCrCuFeNi}$ causes a dilation of the lattice $[29,30]$.

Because of the sluggish diffusion in HEAs, their recrystallization kinetics are slow and annealed cold-rolled material can be very fine grained [31, 32, 33] and contain numerous nanotwins [6-8] (due to the very low stacking fault energy). The sluggish diffusion kinetics can also result in nanosized precipitates [29, 34].

An interesting and challenging question is whether the high configurational entropy present in alloys containing five or more elements is needed to form a single-phase alloy. $\mathrm{Wu}$ et al. [35] explored whether combinations of the component elements of the single-phase fcc quninary HEA FeNiCoCrMn were also single-phase fcc. They cast quaternary, ternary and binary alloys of all possible combinations of the five constituent elements and found that only the quaternary FeNiCoCr, FeNiCoMn and NiCoCrMn, the 
ternary alloys FeNiCo, FeNiCr, FeNiMn, FeCoCr and NiCoMn, and the binary alloys FeNi and NiCo (and, of course pure Ni) were single phase fcc. All the other combinations were either multiphase or had a different crystal structure. They subsequently concluded that the ability to form a single phase was not only determined by the number of elements but also by the type of alloying element.

Additionally, it is worth noting that in many HEA alloys containing more than one phase, some of the phases themselves can be high entropy, as shown in Figure 4 which shows bright field transmission electron microscopy (TEM) images of the HEA $\mathrm{Fe}_{28} \mathrm{Ni}_{18} \mathrm{Mn}_{33} \mathrm{Al}_{13} \mathrm{Cr}_{8}$ (in at. \%). It is clear that this HEA consists of both fcc and $\mathrm{B} 2$ (ordered bcc) phases. Interestingly, based on the energy dispersive X-ray data (in at. \% from five measurements) in Table I, the fcc phase is itself a high entropy phase [36]. The implication is that one can manipulate and optimize the composite microstructure. Since high entropy phases are expected to be stable at high temperatures, the composite may find places for niche applications.

Another interesting phenomenon is that phase separations have often been observed in HEAs [37], for example, in AlCoCrCuFeNi [38] and AlCoCrFeNi [39]. The second phase particles in these HEAs have a size of the order of tens of nanometers with crystal structures and chemical compositions distinct from the matrix phases. Even for some HEAs that appear to be single phase in X-ray diffraction patterns and low-magnification TEM images, atomic-scale structural and chemical characterizations by high-resolution TEM and atom probe tomography often reveal nano-scale phase separation caused by local chemical ordering [40]. For example, all the X-ray diffraction peaks of the $\mathrm{CoCrFeCuNiAl}_{0.5}$ HEA can be indexed as a single fcc phase. However, under high-resolution TEM, the atomic image taken using a high angle annular dark field detector shows obvious structural and chemical variations (Fig.5a). A higher magnification image discloses the local structure ordering with a $L 1_{2}$ domain, which is 
surrounded by a simple fcc phase (Fig. 5b). The $L 1_{2}$ order is further confirmed by the fast Fourier transform pattern shown in Fig. 5c. Local chemical analysis of the imaged region by electron energy loss spectroscopy (EELS) indicates that the $L 1_{2}$ ordered domain is exclusively enriched with $\mathrm{Ni}(\mathrm{FeCoCr}) \mathrm{Al}$ while the simple fcc phase is enriched with $\mathrm{Cu}$ (Fig. 5c). The phase separation, in this case, appears to be triggered by depletion of $\mathrm{Cu}$ from the multicomponent matrix because the formation of the simple fcc phase by $\mathrm{Cu}$ uphill diffusion is kinetically easier than that of the $L 1_{2}$ phase formation, which requires the rearrangements of multiple elements $(\mathrm{Fe}, \mathrm{Cr}, \mathrm{Co}$ and $\mathrm{Ni})$. The observation of phase separation and nanoprecipitation has a profound impact on the potential strengthening of HEAs, especially the creation of favorable $\mathrm{L}_{2}{ }_{2}$ precipitates in an fcc-alloy matrix, parallel to the successful application of $\gamma-\gamma^{\prime}$ Ni-based superalloys. However, the selection of alloy composition, the precipitate-matrix interface structure and chemistry and high temperature stability are all intriguing research subjects for the future study.

\section{Strengthening mechanisms}

Hardening (or strengthening) of crystalline solids occurs when moving dislocations interact with crystalline defects. Depending upon the type of defects, hardening mechanisms are traditionally divided into four categories: solid-solution hardening, which is associated with point defects in the crystal; strain hardening, which is associated with line defects in the crystal; grain boundary hardening, which is associated with planar defects in the crystal; and precipitation and/or dispersion hardening, which is associated with volumetric defects in the crystal. Theories for these four mechanisms have been well developed and are widely documented in standard textbooks. For the convenience of discussion, a summary of these four mechanisms is listed in Table 2. Since each of the above hardening mechanism operates independently, they contribute individually and 
simultaneously to the overall strength of a crystal. The resultant strength of the crystal, $\sigma_{t}$, is, therefore,

$$
\sigma_{t}=\sigma_{f}+\sigma_{s s}+\sigma_{s h}+\sigma_{p t}+\sigma_{g b}
$$

where the subscripts $s s$ stands for solid solution, sh for strain (work) hardening, $p t$ for precipitation/dispersion, $g b$ for grain boundary, and $\sigma_{f}$ represents the intrinsic or frictional strength of the crystal. For high-entropy alloys, there have been limited systematic studies on the above four mechanisms. In the following, the contributions of each of the four mechanisms based on the available data in the literature are summarized.

\section{Strain hardening}

$\mathrm{Lu}$ and coworkers [41] found that, whereas single-phase fcc-FeCrNiCoMn was relatively soft in the as-cast state, cold working significantly increased its strength, as shown in Fig. 6. The result shows that 50\% cold working can enhance the yield strength by a factor of five (from 200MPa to 1000MPa). However, it is also noted in Fig. 6 that plastic instability develops immediately after yielding in samples cold-rolled over $50 \%$, indicating it is at the plastic strain limit of the alloy. Yao et al [42] reported that another single-phase fcc- $\mathrm{Fe}_{40} \mathrm{Mn}_{27} \mathrm{Ni}_{26} \mathrm{Co}_{5} \mathrm{Cr}_{2}$ also exhibited extended ductility and significant strain hardening. For this alloy, plastic instability also occurs right after the yielding when it has been cold-worked $60 \%$. In summary, the above two HEAs behave quite similar to the conventional fcc-metals, which is consistent with the observations of Wu et al [43].

\section{Grain-boundary hardening}

There have been limited reports systematically examining the effect of grain size on the yield strength of HEAs [44, 45]. For example, Liu et al [44] performed cold rolling of a fcc-FeCrNiCoMn alloy to $70 \%$ and then, annealed the cold-rolled samples at $850-950^{\circ} \mathrm{C}$ to produce various grain sizes. Microhardness tests were subsequently carried 
out on the annealed samples and it was found that $k_{\mathrm{HP}}=677 \mathrm{MPa}-(\mu \mathrm{m})^{-1 / 2}$, where $k_{\mathrm{HP}}$ is the Hall-Petch coefficient in the classical equation

$$
H=H_{o}+k_{H P} d^{-1 / 2}
$$

It has been shown [46] that, in the conventional metallic alloys, the upper bound value of $k_{H P}^{f c c}$ is about $600 \mathrm{MPa}-(\mu \mathrm{m})^{-1 / 2}$ and $k_{H P}^{b c c} \approx 3 k_{H P}^{f c c}$. In comparison, the $k_{H P}$ value for the above fcc-FeCrNiCoMn alloy is in line with, and slightly higher than, that for the conventional metallic alloys. A study of grain-size effects was also reported by Wu et al [45] in another HEA. In that study, the authors cold-rolled a fcc-FeCoNiCr alloy to $90 \%$ reduction and, then, annealed it at $900^{\circ} \mathrm{C}$ for various durations of time to produce different grain sizes. Subsequent tensile tests showed that the yield strength well obeyed the Hall-Petch relationship with $k_{\mathrm{HP}}=900 \mathrm{MPa}-(\mu \mathrm{m})^{-1 / 2}$, apparently much higher than that for the fcc-FeCrNiCoMn alloy. The higher $k_{H P}$ was probably due to the fact that the fcc-FeCoNiCr contains a large amount of twins, which can also contribute to the strengthening [45]. In contrast, the annealed fcc-FeCrNiCoMn samples contain a negligible amount of twins [44]. Therefore, we may tentatively conclude that grain refinement is an effective strengthening mechanism in HEAs, comparable to and probably slightly stronger than that observed in conventional fcc-metals. There is no systematical study on the grain size effect in bcc or hcp-HEAs. However, based on the result [46] that $k_{H P}^{b c c} \approx 3 k_{H P}^{f c c}$ in the conventional metals, research along this line is worth pursuing.

\section{Solid-solution hardening}

Intuitively, one would expect a complete atomic intermixing, severe lattice distortion and, consequently, a solid-solution strengthening effect in HEAs. For conventional solid-solution alloys, we have mathematical relationships for the strengthening in dilute 
solid solutions, as derived by Fleischer and co-workers [47]. A general picture for the strengthening is viewed as dislocations moving in a solvent matrix locally distorted by solute atoms. As discussed earlier, it is impossible to differentiate solvent and solute in a HEA.

It is noted that the "intrinsic" strength of a HEA is usually higher than that of the individual constituent elements. For example, the yield strength of fully-annealed pure Ni is about $150 \mathrm{MPa}$, which is lower than that of the fully homogenized fcc-FeCoNiCrMn, which is $200 \mathrm{MPa}$ [43]. Consequently, some efforts have been made to extend the traditional equations for dilute solid solutions or their modified versions to include concentrated alloys, in an attempt to rationalize the increase in strength, that is, the so-called solid-solution effects in HEAs [48, 49]. However, the modeling effort always encounters the inherent problem of identifying the solvent and solutes in the alloys and their interactions.

It is felt that to understand the "intrinsic" strength of a HEA, namely, the first term in Eq.(7), is a problem of its own and requires intensive research. To evaluate the solid-solution effect in HEAs, however, is another matter. In the latter case, the fully homogenized sample forms the "baseline" and the evaluation of solid-solution effect is the study of strength variation after adding foreign atoms to the baseline alloy. Obviously, this area is yet to be explored. Since in a single-phase HEA, atoms are homogeneously spread throughout the alloy and the lattice distortion on the average would be uniform (for lattice distortion, see discussion in section 2), dislocations are anticipated to experience a uniform viscous resistance while moving through lattice. The traditional picture for solid-solution strengthening, in which a distinct local lattice distortion caused by the individual solute, is difficult to visualize.

It is worth noting that Lei and $\mathrm{Lu} \mathrm{[50]} \mathrm{recently} \mathrm{found} \mathrm{that} \mathrm{the} \mathrm{presence} \mathrm{of} \mathrm{interstitial}$ nitrogen in a bcc-HEA caused a dramatic hardening. A quantitative evaluation of the 
effect is currently underway. Based on the traditional wisdom, interstitial solid-solution strengthening is generally much more significant as compared to substitutional strengthening [47].

\section{Precipitation hardening}

In terms of precipitation hardening in HEAs, published results are somewhat sketchy and mysterious. There have been reports on the observation of age hardening in some HEAs, for example, a mixed fcc+bcc $\mathrm{CuCr}_{2} \mathrm{Fe}_{2} \mathrm{NiMn}$ [51], a mixed fcc+bcc $\mathrm{Al}_{0.3} \mathrm{CrFe}_{1.5} \mathrm{MnNi}_{0.5}$ and single-phase bcc- $\mathrm{Al}_{0.5} \mathrm{CrFe}_{1.5} \mathrm{MnNi}_{0.5}$ [52]. The aging temperature is typically around $600-800^{\circ} \mathrm{C}$ and the strength increase is about $2-3$ times. The strengthening phases were identified to be tetragonal $\mathrm{Cr}_{5} \mathrm{Fe}_{6} \mathrm{Mn}_{8}$ phase in bcc- $\mathrm{Al}_{0.5} \mathrm{CrFe}_{1.5} \mathrm{MnNi}_{0.5}$ alloys and $\mathrm{Cr}_{5} \mathrm{Fe}_{6} \mathrm{Mn}_{8}$ ( $\rho$ phase) in $\mathrm{CuCr}_{2} \mathrm{Fe}_{2} \mathrm{NiMn}$. These precipitates were relatively bulky (>micron size), quite different than the fine precipitates $(\sim \mathrm{nm})$ found in the traditional precipitation-hardened alloys. Furthermore, microstructural kinetics such as precipitate size, shape and distribution and the feature of precipitate-matrix interfaces were not carefully characterized; in other words, the nature of age hardening was largely unclear. In this workshop, $\mathrm{He}$ and $\mathrm{Lu}$ [53] reported the observation of significant hardening of a quaternary $\mathrm{Fe}_{1-\mathrm{x}-\mathrm{y}-\mathrm{z}} \mathrm{Co}_{\mathrm{x}} \mathrm{Ni}_{\mathrm{y}} \mathrm{Cr}_{\mathrm{z}} \mathrm{HEA}$ by adding $\mathrm{Ti}$ and $\mathrm{Al}$, which leads to phase separation and subsequent precipitation of fine precipitates $(\sim 10 \mathrm{~nm})$. As shown in Fig. 7, the base alloy is relatively soft in the homogenized state (yield strength $<200 \mathrm{MPa}$ ), similar to a regular fcc-alloy. However, after aging at $650^{\circ} \mathrm{C}$, the alloy exhibits a yield strength of over $1 \mathrm{GPa}$ and still keeps a respectable tensile ductility of over $15 \%$. This result is phenomenal, but it also raises a series of questions: 1) What is the nature of the nanoprecipitates? 2) Is the precipitate/matrix interface coherent?

3) Are these nanoprecipitates thermally stable? From the viewpoint of good potential for high-temperature applications, additional research to answer these questions is certainly 
warranted.

\section{High-temperature mechanical properties}

As discussed earlier, HEAs have four intrinsic core effects: high entropy, slow diffusion, lattice distortion, and cocktail effects. Incidentally, all these effects favor high temperature applications. For example, high entropy enhances phase and microstructural stability, especially at high temperatures. Slow diffusion retards structure recovery, and thus improves high temperature resistance. Lattice distortion leads to high friction for dislocation motion. Based on these attributes, preliminary research work has been reported in literature.

Senkov et al. firstly characterized the compressive properties of a few bcc HEAs, including TaNbHfZrTi[54], NbMoTaW and VNbMoTaW[55], at elevated temperatures. Microstructural characterization of the fractured VNbMoTaW HEA specimens after compression deformation at $800^{\circ} \mathrm{C}$ indicated that no appreciable microstructural change had occurred. More importantly, the yield strength of the NbMoTaW and VNbMoTaW HEAs above $800^{\circ} \mathrm{C}$ was around $600 \sim 800 \mathrm{MPa}$ and $400 \sim 450 \mathrm{MPa}$ at $1600^{\circ} \mathrm{C}$, much superior to that of the traditional superalloys Inconel 718 and Haynes 230, confirming that these refractory bcc HEAs have a strong resistance to high-temperature softening.

For fcc HEAs, some high-temperature studies have also been carried out, mainly on the typical fcc FeCoNiCrMn $[56,57]$. In this case, long-term annealing data revealed that even after one month aging at $950{ }^{\circ} \mathrm{C}$, the typical $\mathrm{FeCoNiCrMn}$ alloy remained a single phase with no precipitation of any second phase[58], indicating good microstructural and phase stability.

Additionally, the steady-state flow behavior of the FeCoNiCrMn HEA at temperatures from $750-850^{\circ} \mathrm{C}$ was also characterized via strain-rate jump tests [59]. 
Mechanistically, the steady-state stress - strain rate relationship can be well described by either dislocation climb in the high strain rate regime or dislocation glide in the low strain rate regime, as shown in Fig. 8.

Tensile tests of this fcc HEA with different grain sizes [57] at different temperatures were also systematically performed and the results indicated that the high-temperature strength of this alloy was not particularly high. It may not be a surprise since monolithic pure metals lack strengthening mechanisms at high temperatures. To improve the high temperature strength, precipitates or second-phase particles must be introduced into the matrix to form barriers to the dislocation motion. From the high-temperature tension study, Otto et al.[57] also observed various $\mathrm{Mn}$ and Cr-riched precipitates form, especially in samples that deformed at a higher temperatures and lower strain rates, presumably from a prolonged thermal exposure. From these limited studies, we may tentatively conclude that further study of precipitation or dispersion hardened HEAs is certainly merited.

High-performance heat resistant alloys should simultaneously possess several key properties: excellent mechanical strength, resistance to thermal creep deformation, good surface stability and resistance to corrosion or oxidation. Therefore, in the development of HEAs for high-temperature applications, several challenges and issues lie ahead and need to be addressed; this is discussed in the next section. 


\section{Perspectives and Future Prospects}

High entropy alloys constitute a new class of materials, quite different from the traditional alloys, consisting of several principal elements intermixed in the crystal lattice. They are often perceived as solid solution alloys but without clear identification of solvent and solutes. Thus, it is difficult to apply current theories of solid-solution strengthening mechanisms to HEAs. Solute strengthening models take into account several effects including: the lattice strain due to the solute atoms; the modulus change due to the solute; electrical effects; and the effects of the solute on the stacking fault energy. Short range ordering can also occur and lead to strengthening. In HEAs where there are five or more elements present in similar proportions, the idea that a particular element can be considered to be a solute does not make sense. In addition, the crystallographic lattice of HEAs containing multiple primary elements with different atomic sizes and chemical properties is severely distorted, and in such a distorted lattice, the creation, interaction and movement of all kinds of crystal defects, e.g. vacancy, dislocation, stacking fault and grain boundary, etc., are expected to be different from those in the conventional alloys. Novel characterization techniques, advanced computation methods, and extensive experimental work are needed so that the relationship between microstructure and mechanical properties can be established in these concentrated alloys.

We believe that the characterization of lattice distortion and its effects on defect energies is important for the understanding of strengthening in HEAs. However, to define lattice distortion, measure lattice distortion, and calculate the lattice distortion energy are still challenging tasks at this moment. Moreover, developing suitable mechanisms and theories to correlate various phenomena with lattice distortion are needed to be built through a combination of both theoretical and experimental work. 
Strain and strain energy might be used to describe the degree of topological distortion in the whole-solute lattice. Because vacancies, dislocations, stacking faults, twins, and grain boundaries are all formed in the lattice, the atomic configuration of these defects would be different between an unalloyed lattice without distortion and the whole-solute lattice with distortion. As a result, all defect energies as conventionally given in the pure metals or diluted alloy needs to be re-checked in such severely distorted lattices.

In conventional alloys, segregation to line defects and planar defects occurs in order to lower the overall energy. Segregation in general reduces the mobility of dislocations, the cross-slip ability of screw dislocations under applied stress, and the mobility of boundaries during grain growth. Hence, whether or not segregation occurs to dislocations, stacking faults and grain boundaries in HEAs is an important issue to be clarified. Certainly, measurements of weak beam images of dislocations in the TEM, and examination of X-ray and electron diffraction patterns can provide useful information in this regard. No matter whether segregation occurs or not, a distorted lattice with higher energy level and a larger lattice friction already has a large effect on the mobility of dislocations, cross-slip ability of screw dislocations, and twinning tendency which influence deformation behaviors and, thus, the deformation structure. For these dynamic behaviors, solid solution hardening, serration behavior, grain size strengthening or Hall-Petch equation, twinning-induced strain hardening, ductility, creep, and fatigue initiation and propagation all need to be assessed and investigated in the future.

As for physical properties, the topological lattice distortion will influence the lattice constant, diffusion scattering of X-ray diffraction, melting temperature, electron mobility, electrical conductivity, thermal conductivity, temperature coefficient, and damping capacity. In addition, as mentioned in Section 2, physicochemical lattice distortion will also influence diffusion scattering, electron mobility, electrical conductivity, thermal 
conductivity, temperature coefficient, and damping capacity.

From the viewpoint of high temperature applications, developed HEAs must have the following attributes.

1) Reduced lattice diffusion

Lattice diffusion is one of the key factors determining the high-temperature deformation behavior, and the bcc structure generally has a relatively higher self-diffusion rate than the close-packed fcc and hcp structures. Slowing down the lattice diffusion is important to maintain high phase stability and achieve excellent creep properties.

2) Effective strengthening mechanisms

A monolithic simple-phase alloy, either bcc or fcc, is not strong enough at high temperatures and an effective strengthening mechanism need to be utilized. Among all the possible strengthening mechanisms introduced earlier, precipitation hardening is probably the most promising method for high-temperature strengthening, in a manner similar to that in Ni-based superalloys and heat resistant steels. In addition, grain boundaries often tend to be weak regions at high temperatures, a feature which needs to be properly controlled, especially when the service temperature is high (usually above $0.5 \mathrm{Tm})$. Intensive efforts should also be devoted to characterize and understand the creep deformation behavior and the underlying mechanisms.

3) Improved oxidation performance

As discussed above, refractory bcc HEAs showed strong resistance to high-temperature softening and substantial microstructural stability. However, their oxidation performance, especially under long-time heat exposure (e.g., creep condition) needs to be improved. In this regard, fcc HEAs are much better because of the presence of anti-oxidation elements like $\mathrm{Cr}, \mathrm{Al}$, or $\mathrm{Si}$. 
4) Cost reduction

Considering the fact that HEAs usually possess equal (or near) molar ratios of components, the cost is an inevitable problem if expensive raw elements such as $\mathrm{Nb}, \mathrm{Hf}$, Ta, W, Zr and V are present. Relatively speaking, fcc HEAs containing Fe, Ni, Cr, Mn and $\mathrm{Al}$ are much cheaper. Cost reduction from both raw materials and processing with no loss of high-temperature performance is also a challenge for widespread uses of HEAs.

In the above, we have reported the current understanding of the microstructure-mechanical property correlation in HEAs based on the discussion in this workshop, which consists of the definition, core characteristics, atomic size effects, microstructure, strengthening mechanisms and high-temperature deformation behavior. It by no means includes all important and challenging issues. The perspective for future research associated with the development of HEAs was also summarized. Our analysis and discussion suggest that extensive work, both theoretical and experimental, is urgently needed to fully understand the microstructure-properties relationship of this novel family of metallic materials. It is our hope that this report would stimulate the interests of scientists and engineers to work together, both in theory and experiment, to develop this novel family of metallic materials.

\section{Acknowledgments}

The financial support from National Natural Science Foundation of China (No. 51010001), 111 Project (B07003) and Program for Changjiang Scholars and Innovative Research Team (IRT_14R05) is greatly acknowledge. The authors also would like to thank the workshop organizing committee member, Drs. X. J. Liu, Y. Wu, Mrs. J. Y. He, Z. F. Lei and Ms H. L. Huang. Fruitful discussion with workshop attendees, Profs. C. Dong, K. F. Yao, J. J. Kai, Dr. M. H. Tsai and Mr. X. D. Xu, are also greatly appreciated. IB was supported by U.S. Department of Energy (DOE), Office of Basic Energy Sciences grant DE-FG02-07ER46392. TGN was supported by U.S. NSF grant DMR-0905979. 


\section{References}

[1] Cantor B, Chang ITH, Knight P, Vincent AJB. Microstructural development in equiatomic multicomponent alloys. Mater Sci Eng A 2004;375-377:213-8.

[2] Yeh JW, Chen SK, Lin SJ, Gan JY, Chin TS, Shun TT, Tsau CH, Chang SY. Nanostructured high-entropy alloys with multiple principal elements: novel alloy design concepts and outcomes. Adv Eng Mater 2004;6:299-303.

[3] Cantor B. Multicomponent and high entropy alloys. Entropy 2014;16(9):4749-4768.

[4] Miracle DB, Miller JD, Senkov ON, Woodward C, Uchic MD, Tiley J. Exploration and development of high entropy alloys for structural applications. Entropy 2014;16(1):494-525.

[5] Tsai MH, Yeh JW. High-entropy alloys: a critical review. Mater Res Lett 2014;2(3):107-123.

[6] Zhang Y, Zuo TT, Tang Z, Gao MC, Dahmen KA, Liaw PK, Lu ZP. Microstructures and properties of high-entropy alloys. Prog Mater Sci 2014;61:1-93.

[7] Yeh JW. "Recent Progress in High-entropy Alloys". Ann Chim Sci Mat 2006;31(6):633-648.

[8] Yeh JW. "Alloy Design Strategies and Future Trends in High-Entropy Alloys". JOM 2013;65(12):1759-1771.

[9] Tsai KY, Tsai MH, Yeh JW. Sluggish diffusion in Co-Cr-Fe-Mn-Ni high-entropy alloys. Acta Mater 2013;61(13):4887-4897.

[10] Yeh JW, Chang SY, Hong YD, Swe-Kai C, Su-Jien L. Anomalous decrease in X-ray diffraction intensities of $\mathrm{Cu}-\mathrm{Ni}-\mathrm{Al}-\mathrm{Co}-\mathrm{Cr}-\mathrm{Fe}-\mathrm{Si}$ alloy systems with multi-principal elements. Mater Chem Phys 2007;103(1):41-46.

[11] Hume-Rothery W, Coles BR. The transition metals and their alloys. Adv Phys 1954;3(10):149-242.

[12] Eshelby JD. The continuum theory of lattice defects. Solid State Phys 1956;3:79-144.

[13] Zhang Y, Zhou YJ, Lin JP, Chen GL, Liaw PK. Solid - Solution Phase Formation Rules for Multi - component Alloys. Adv Eng Mater 2008;10(6):534-538.

[14] Guo S, Hu Q, Ng C, Liu CT. More than entropy in high-entropy alloys: Forming solid solutions or amorphous phase. Intermetallics 2013;41:96-103.

[15] Guo S. Phase selection rules for cast high entropy alloys: an overview. Mater Sci Technol 2015.

[16] Takeuchi A, Amiya K, Wada T, Yubuta K, Zhang W, Makino A. Entropies in Alloy Design for High-Entropy and Bulk Glassy Alloys. Entropy 2013;15:3810-3821.

[17] Guo S, Ng C, Wang Z, Liu CT. Solid solutioning in equiatomic alloys: Limit set by topological instability. J Alloys Compd 2014;583:410-413.

[18] Pusey P. The effect of polydispersity on the crystallization of hard spherical colloids. J Physique 1987:48(5):709-712.

[19] Wang Z, Huang Y, Yang Y, Wang J, Liu CT. Atomic-size effect and solid solubility of multicomponent alloys. Scr Mater 2015;94:28-31.

[20] Egami T. Universal criterion for metallic glass formation. Mater Sci Eng A 1997;226:261-267. 
[21] Miracle DB, Sanders WS, Senkov ON. The influence of efficient atomic packing on the constitution of metallic glasses. Philos Mag 2003;3(20):2409-2428.

[22] Wang ZJ, Qiu WF, Yang Y, Liu CT. Atomic-size effects in crystalline lattices with multiple principal elements. Intermetallics 2015;64:63-69

[23] Ding HY, Yao KF. High entropy Ti20Zr20Cu20Ni20Be20 bulk metallic glass. J Non-Cryst Solids 2013;364:9-12.

[24] Ding HY, Shao Y, Gong P, Li JF, Yao KF. A senary TiZrHfCuNiBe high entropy bulk metallic glass with large glass-forming ability. Mater Lett 2014;125:151-153.

[25] Huo J, Huo L, Men H, Wang X, Inoue A, Wang J, Chang C, Li RW. The magnetocaloric effect of Gd-Tb-Dy-Al-M ( $\mathrm{M}=\mathrm{Fe}, \mathrm{Co}$ and $\mathrm{Ni})$ high-entropy bulk metallic glasses. Intermetallics 2015;58:31-35.

[26] Takeuchi A, Amiya K, Wada T, Yubuta K, Zhang W. High-Entropy Alloys with a Hexagonal Close-Packed Structure Designed by Equi-Atomic Alloy Strategy and Binary Phase Diagrams. JOM 2014;66(10):1984-1992.

[27] Takeuchi A, Wang J, Chen N, Zhang W, Yokoyama Y, Yubuta K, Zhu S. Al 0.5 TiZrPdCuNi High-Entropy (HE) Alloy Developed through $\mathrm{Ti}_{20} \mathrm{Zr}_{20} \mathrm{Pd}_{20} \mathrm{Cu}_{20} \mathrm{Ni}_{20}$ HE Glassy Alloy Comprising Inter-Transition Metals. Mater Trans 2013;54(5):776-782.

[28] Wang WH. High-Entropy Metallic Glasses. JOM 2014;66(10):2067-2077.

[29] Tong CJ, Chen YL, Yeh JW, Lin SJ, Chen SK, Shun TT, Tsau CH, Chang SY. Microstructure characterization of $\mathrm{Al}_{\mathrm{x}} \mathrm{CoCrCuFeNi}$ high-entropy alloy system with multiprincipal elements. Metall Mater Trans A 2005;36:881-893.

[30] He JY, Liu WH, Wang H, Wu Y, Liu XJ, Nieh TG, Lu ZP. Effects of Al addition on structural evolution and tensile properties of the FeCoNiCrMn high-entropy alloy system. Acta Mater 2013;62:105-113.

[31] Park N, Watanabe I, Terada D, Yokoyama Y, Liaw PK, Tsuji N. Recrystallization Behavior of CoCrCuFeNi High-Entropy Alloy. Metall Mater Trans A 2014;46:1481-1487.

[32] Bhattacharjee PP, Sathiaraj GD, Zaid M, Gatti JR, Lee C, Tsai CW, Yeh JW. Microstructure and texture evolution during annealing of equiatomic CoCrFeMnNi high-entropy alloy. J Alloys Compd 2014;587:544-552.

[33] Otto F, Hanold NL, George EP. Microstructural evolution after thermomechanical processing in an equiatomic, single-phase $\mathrm{CoCrFeMnNi}$ high-entropy alloy with special focus on twin boundaries. Intermetallics 2014;54:39-48.

[34] Tsai MH, Yuan H, Cheng GM, Xu WZ, Tsai, KY, Tsai CW, Jian WW, Juan CC, Shen WJ, Chuang MH, Yeh JW. Morphology, structure and composition of precipitates in $\mathrm{Al}_{0.3} \mathrm{CoCrCu}_{0.5} \mathrm{FeNi}$ high-entropy alloy. Intermetallics 2013;32: 329-336.

[35] Wu Z, Bei H, Otto F,Pharr GM, George EP. Recovery, recrystallization, grain growth and phase stability of a family of FCC-structured multi-component equiatomic solid solution alloys. Intermetallics 2014;6:131-140.

[36] Meng F, Baker I. unpublished work.

[37] Singh S, Wanderka N, Kiefer K, Siemensmeyer K, Banhart J. Effect of decomposition of the 
$\mathrm{Cr}-\mathrm{Fe}-\mathrm{Co}$ rich phase of $\mathrm{AlCoCrCuFeNi}$ high entropy alloy on magnetic properties.

Ultramicroscopy 2011;111(6):619-622.

[38] Pradeep KG, Wanderka N, Choi P, Banhart J, Murty BS, Raabe D. Atomic-scale compositional characterization of a nanocrystalline $\mathrm{AlCrCuFeNiZn} \mathrm{high-entropy} \mathrm{alloy} \mathrm{using}$ atom probe tomography. Acta Mater 2013;61(12):4696-4706.

[39] Manzoni A, Daoud H, Völkl R, Glatzel U, Wanderka N. Phase separation in equiatomic AlCoCrFeNi high-entropy alloy. Ultramicroscopy 2013;132:212-215.

[40] Xu XD, Liu P, Guo S, Hirata A, Fujita T, Nieh TG, Liu CT, Chen MW. Nanoscale phase separation in a fcc-based $\mathrm{CoCrCuFeNiAl}{ }_{0.5}$ high-entropy alloy. Acta Mater 2015;84:145-152.

[41] Liu WH, He JY, Nieh TG, Lu ZP. unpublished work.

[42] Yao MJ, Pradeep KG, Tasan CC, Raabe D. A novel, single phase, non-equiatomic FeMnNiCoCr high-entropy alloy with exceptional phase stability and tensile ductility. Scr Mater 2014;72:5-8.

[43] Wu Y, Liu WH, Wang XL, Ma D, Stoica AD, Nieh TG, He ZB, Lu ZP. In-situ neutron diffraction study of deformation behavior of a multi-component high-entropy alloy. Appl Phys Lett 2014;104(5):051910.

[44] Liu WH, Wu Y, He JY, Nieh TG, Lu ZP. Grain growth and the Hall-Petch relationship in a high-entropy FeCrNiCoMn alloy. Scr Mater 2013;68(7):526-529.

[45] Wu Z, Bei H, Pharr GM, George EP. Temperature dependence of the mechanical properties of equiatomic solid solution alloys with face-centered cubic crystal structures. Acta Mater 2014;81:428-441.

[46] Wu D, Zhang JY, Huang JC, Bei H, Nieh TG. Grain-boundary strengthening in nanocrystalline chromium and the Hall-Petch coefficient of body-centered cubic metals. Scr Mater 2013;68(2):118-121.

[47] Fleischer RL. The flow stress of body-centered cubic metals: inherent lattice hardening or solution hardening?. Acta Metall 1967;15(9):1513-1519.

[48] Senkov ON, Scott JM, Senkova SV, Miracle DB, Woodward CF. Microstructure and room temperature properties of a high-entropy TaNbHfZrTi alloy. J Alloy Compd 2011;509(20):6043-6048.

[49] Toda-Caraballo I, Rivera-Díaz-del-Castillo PEJ. Modelling solid solution hardening in high entropy alloys. Acta Mater 2015;85:14-23.

[50] Lei ZF, Wu Y, He JY, Huang HL, Nieh TG, Lu ZP. unpublished work.

[51] Ren B, Liu ZX, Cai B, Wang MX, Shi L. Aging behavior of a $\mathrm{CuCr}_{2} \mathrm{Fe}_{2} \mathrm{NiMn}$ high-entropy alloy. Mater Des 2012;33:121-126.

[52] Chen ST, Tang WY, Kuo YF, Chen SY, Tsau CH, Shun TT, Yeh JW. Microstructure and properties of age-hardenable $\mathrm{Al}_{\mathrm{x}} \mathrm{CrFe}_{1.5} \mathrm{MnNi}_{0.5}$ alloys. Mater Sci Eng A 2010;527(21):5818-5825.

[53] He JY, Huang HL, Wu Y, Nieh TG, Lu ZP. unpublished work.

[54] Senkov ON, Scott JM, Senkova SV, Meisenkothen F, Miracle DB, Woodward CF. "Microstructure and elevated temperature properties of a refractory TaNbHfZrTi alloy." J 
Mater Sci 2012;47(9):4062-4074.

[55] Senkov ON, Wilks GB, Scott JM, Miracle DB. "Mechanical properties of $\mathrm{Nb}_{25} \mathrm{Mo}_{25} \mathrm{Ta}_{25} \mathrm{~W}_{25}$ and $\mathrm{V}_{20} \mathrm{Nb}_{20} \mathrm{Mo}_{20} \mathrm{Ta}_{20} \mathrm{~W}_{20}$ refractory high entropy alloys." Intermetallics 2011;19(5):698-706.

[56] Gali A, George EP. "Tensile properties of high- and medium-entropy alloys." Intermetallics 2013;39:74-78.

[57] Otto F, Dlouhý A, Somsen C, Bei H, Eggeler G, George EP. "The influences of temperature and microstructure on the tensile properties of a $\mathrm{CoCrFeMnNi}$ high-entropy alloy." Acta Mater 2013;61(15):5743-5755.

[58] Liu WH, Lu ZP. unpublished work.

[59] He JY, Zhu C, Zhou DQ, Liu WH, Nieh TG, Lu ZP. "Steady state flow of the FeCoNiCrMn high entropy alloy at elevated temperatures." Intermetallics 2014;55:9-14. 


\section{TABLES}

Table 1

\begin{tabular}{|l|l|l|l|l|l|}
\hline phase & Fe & Ni & Mn & Al & Cr \\
\hline fcc & $36.7 \pm 0.2$ & $10.0 \pm 0.7$ & $35.5 \pm 0.5$ & $9.7 \pm 0.3$ & $8.1 \pm 0.1$ \\
\hline B2 & $4.6 \pm 0.2$ & $40.3 \pm 0.3$ & $17.1 \pm 0.8$ & $37.5 \pm 0.2$ & $0.7 \pm 0.1$ \\
\hline
\end{tabular}


Table 2

\begin{tabular}{c|cccc}
\hline \multicolumn{2}{c|}{$\begin{array}{c}\text { Solid-Solution } \\
\text { Hardening }\end{array}$} & Strain Hardening & $\begin{array}{c}\text { Grain-Boundary } \\
\text { Hardening }\end{array}$ & $\begin{array}{c}\text { Precipitation or } \\
\text { Dispersion } \\
\text { Hardening }\end{array}$ \\
\hline Sources & Solute conc., $c$ & $\begin{array}{c}\text { Dislocation } \\
\text { density, } \rho\end{array}$ & Grain size, $d(\mu \mathrm{m})$ & Particle radius, $r$ \\
Effect & $\left(\frac{G \varepsilon_{s}^{3 / 2} c^{1 / 2}}{700}\right)^{*}$ & $\alpha G b \sqrt{\rho}$ & $k_{y} d^{-1 / 2}$ & $\frac{2 \pi r \gamma_{s}}{b L}$ (cutting) \\
\cline { 2 - 5 } & & & $\frac{G b}{L-2 r}$ (bowing) \\
\hline
\end{tabular}

$G$ : shear modulus, $b$ : Burgers vector, $\gamma_{s}$ : precipitate-matrix interface energy

$* \varepsilon_{s} \approx\left|\frac{\varepsilon_{G}}{1+\frac{1}{2}\left|\varepsilon_{G}\right|}-3 \varepsilon_{a}\right|$, where $\varepsilon_{a}=\frac{1}{a} \frac{d a}{d c}$ is the lattice mismatch and $\varepsilon_{G}=\frac{1}{G} \frac{d G}{d c}$ is the modulus mismatch. 


\section{Figures}

\section{Figure 1}

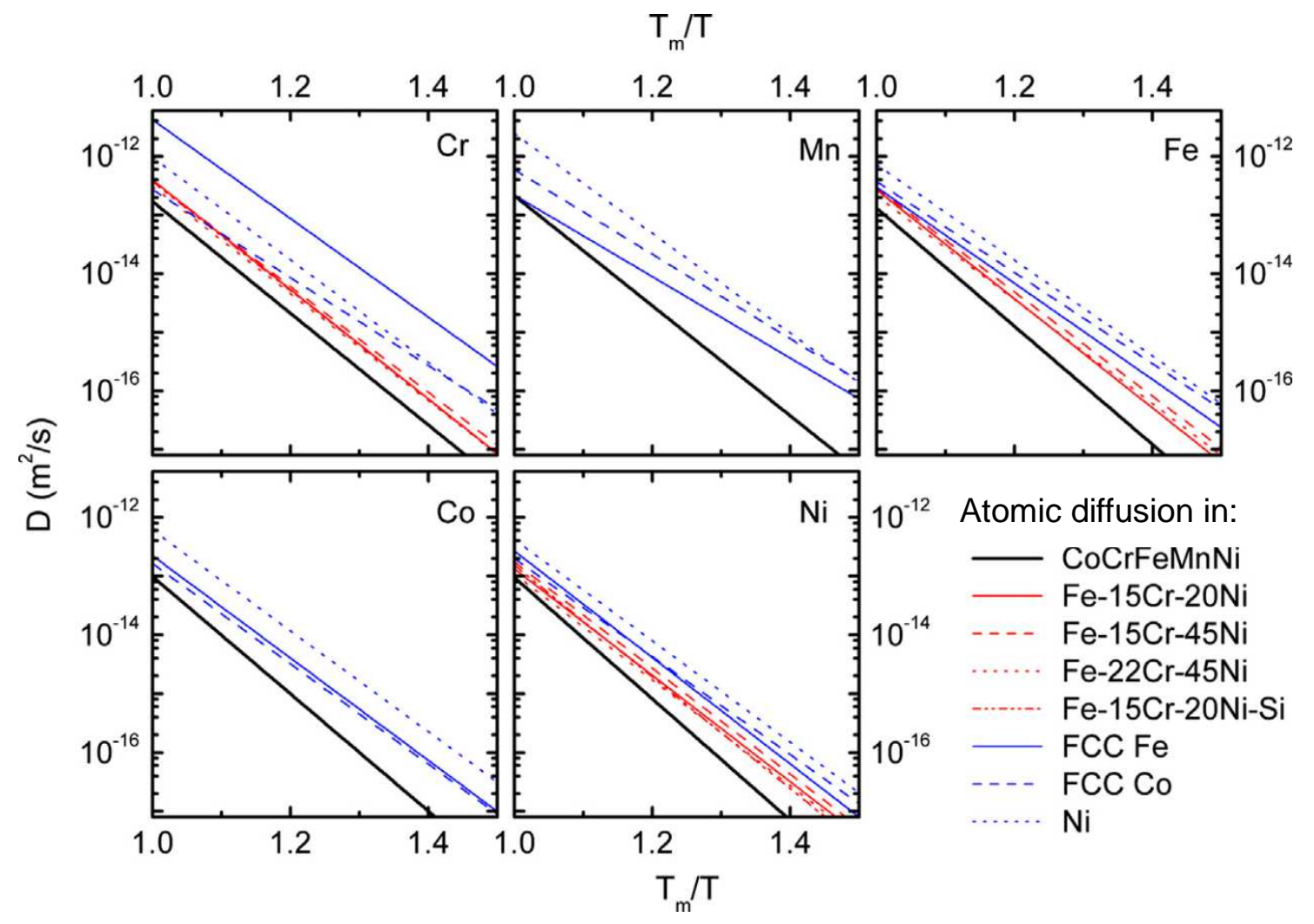


Figure 2

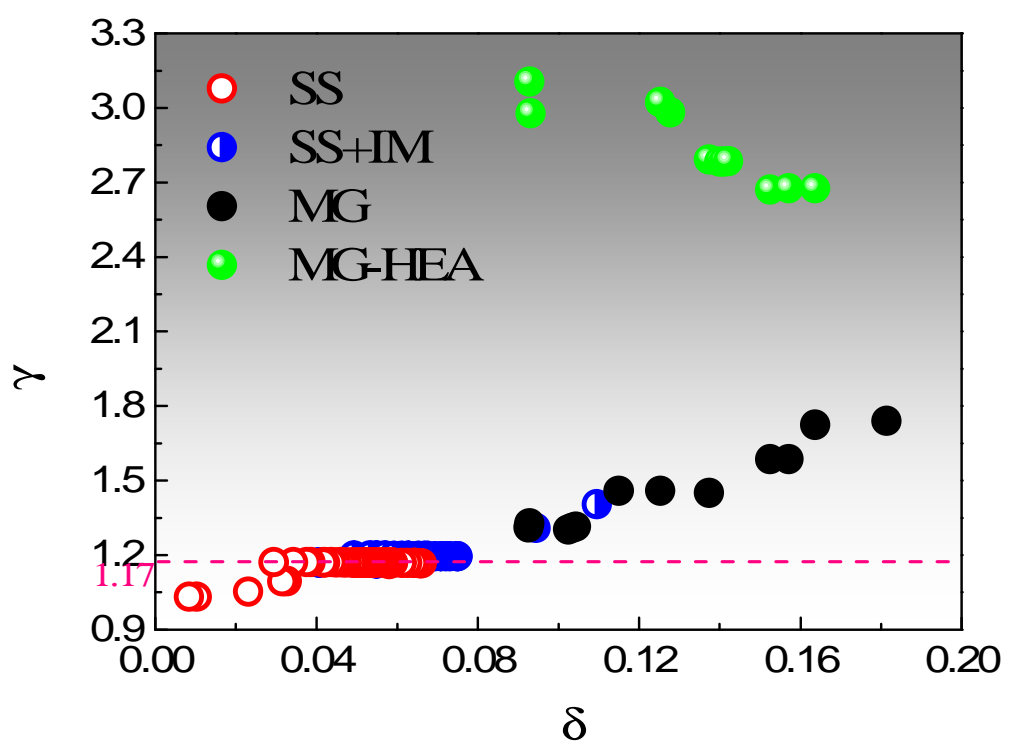


Figure 3
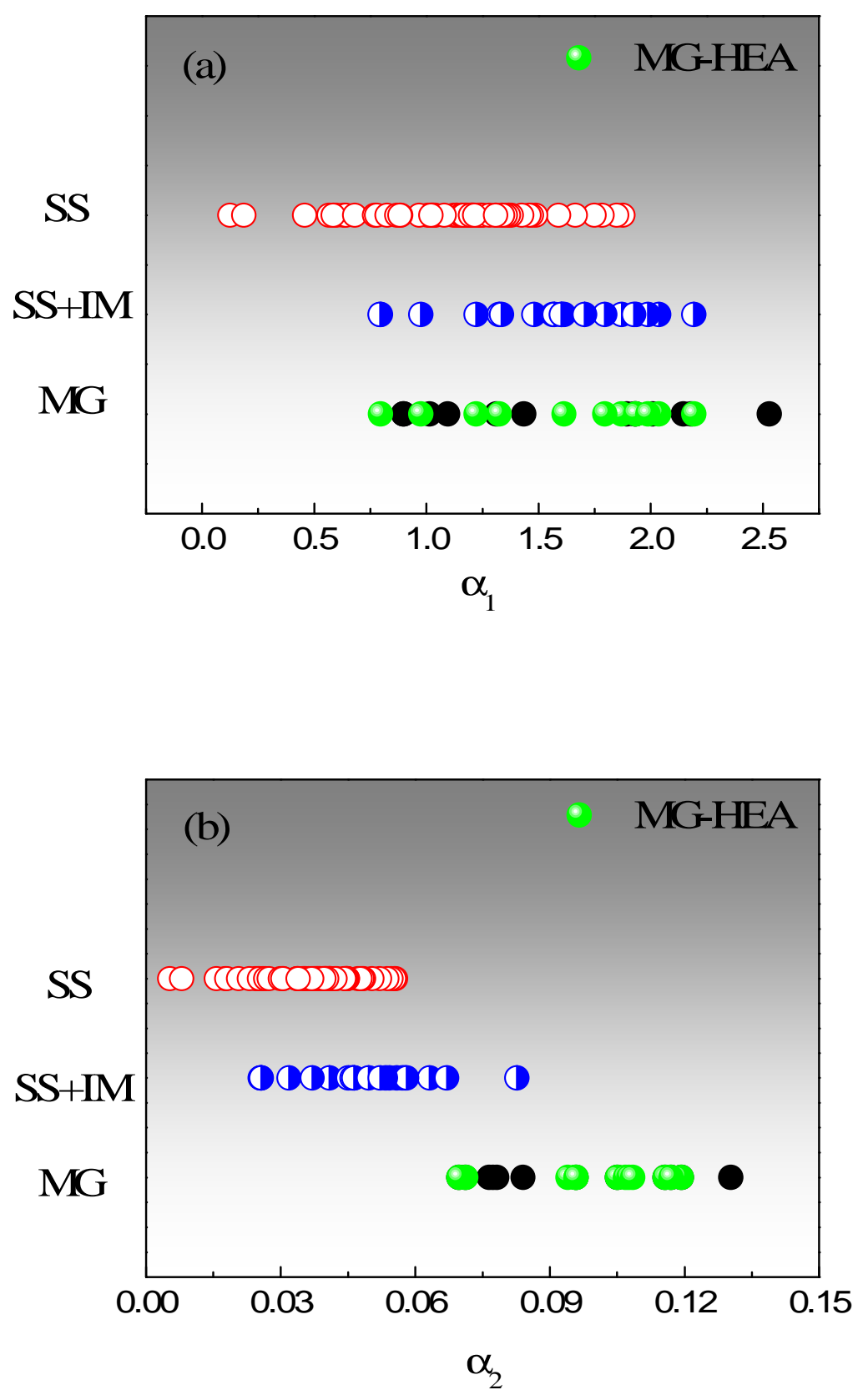
Figure 4

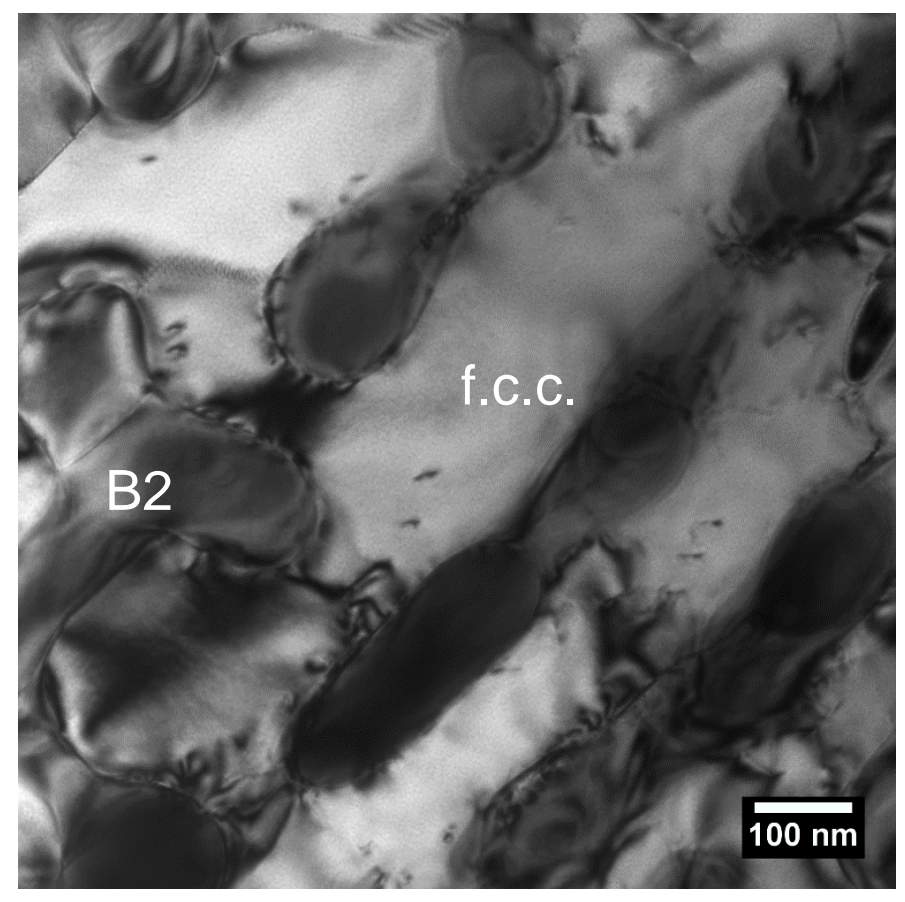


Figure 5

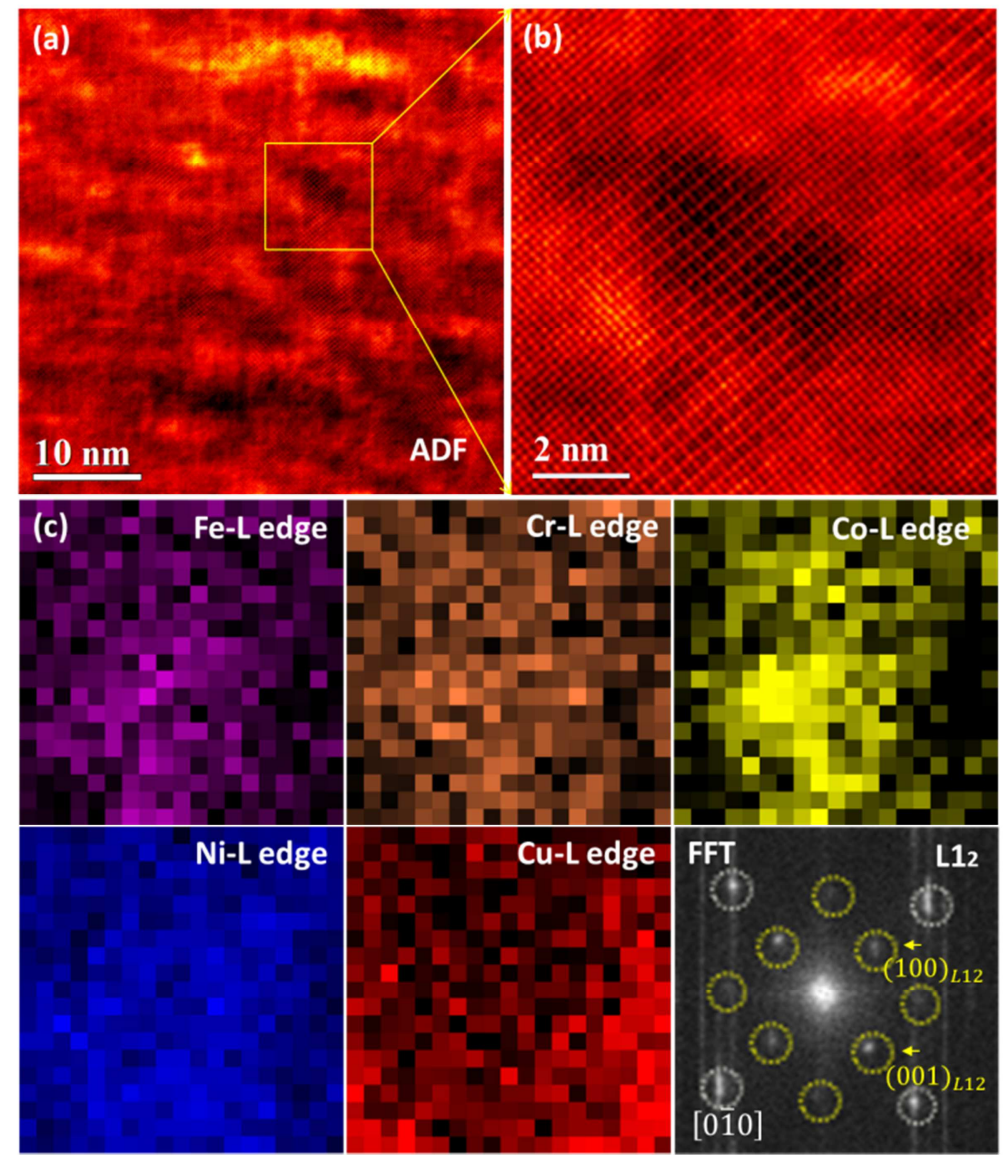


Figure 6

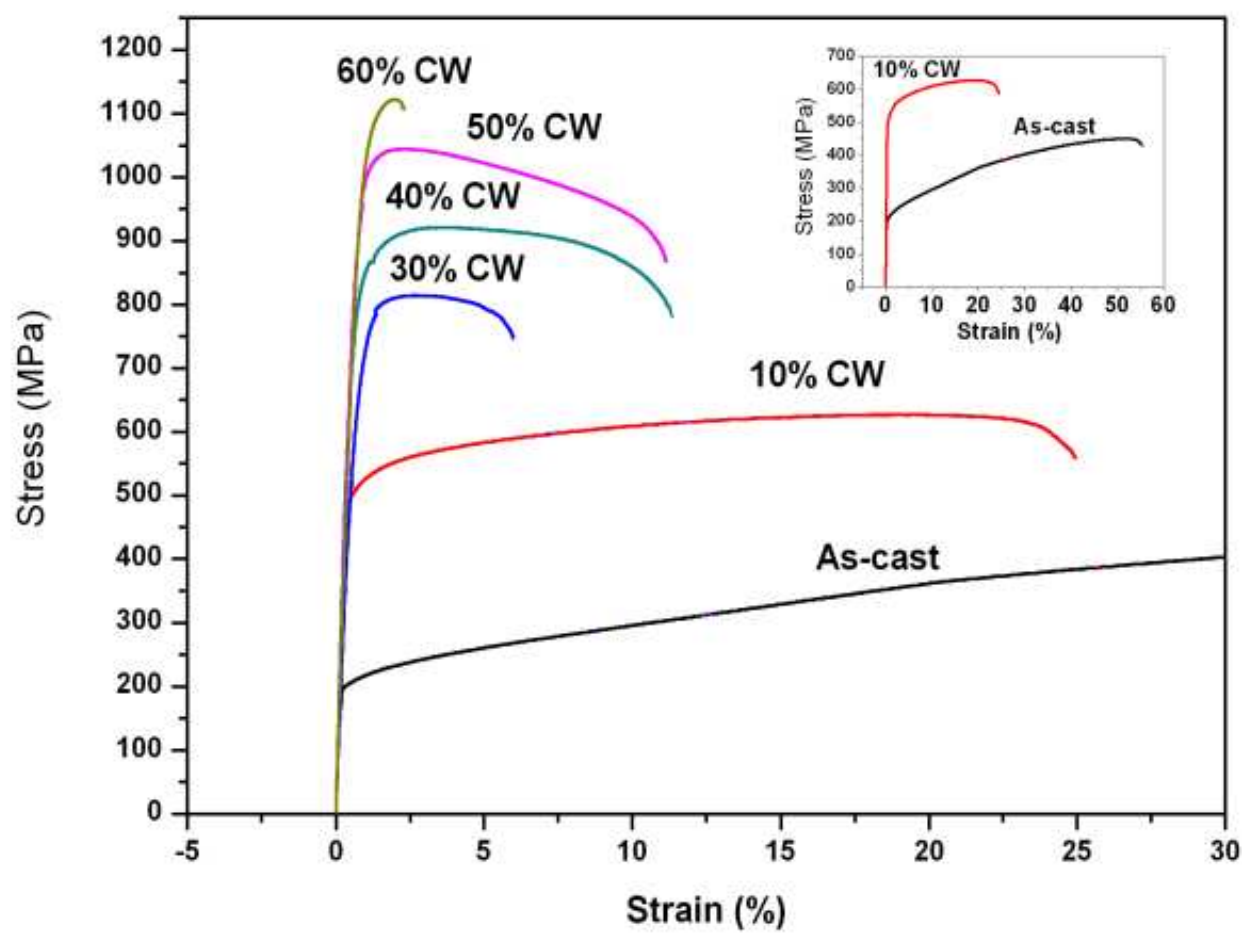


Figure 7

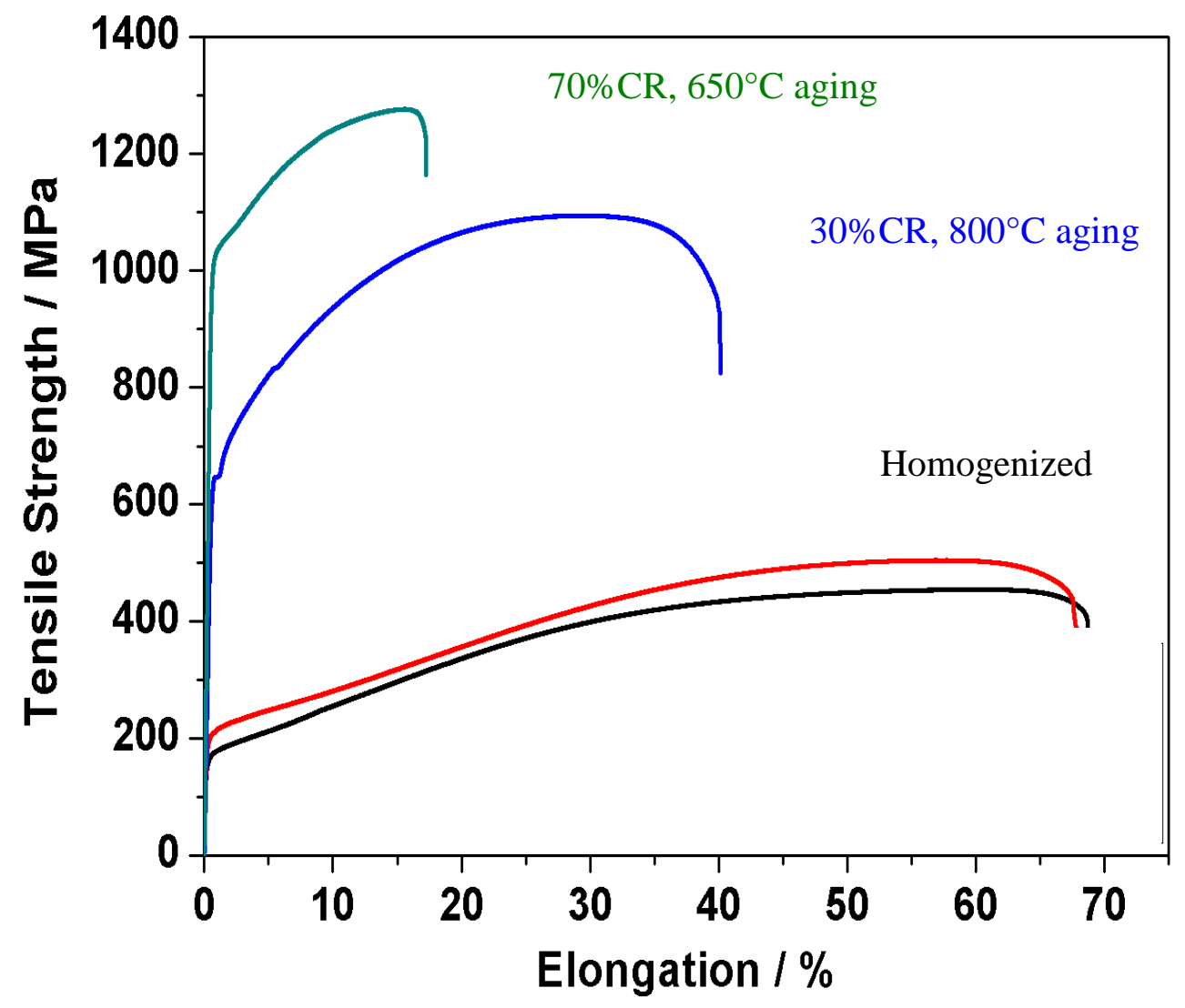


Figure 8

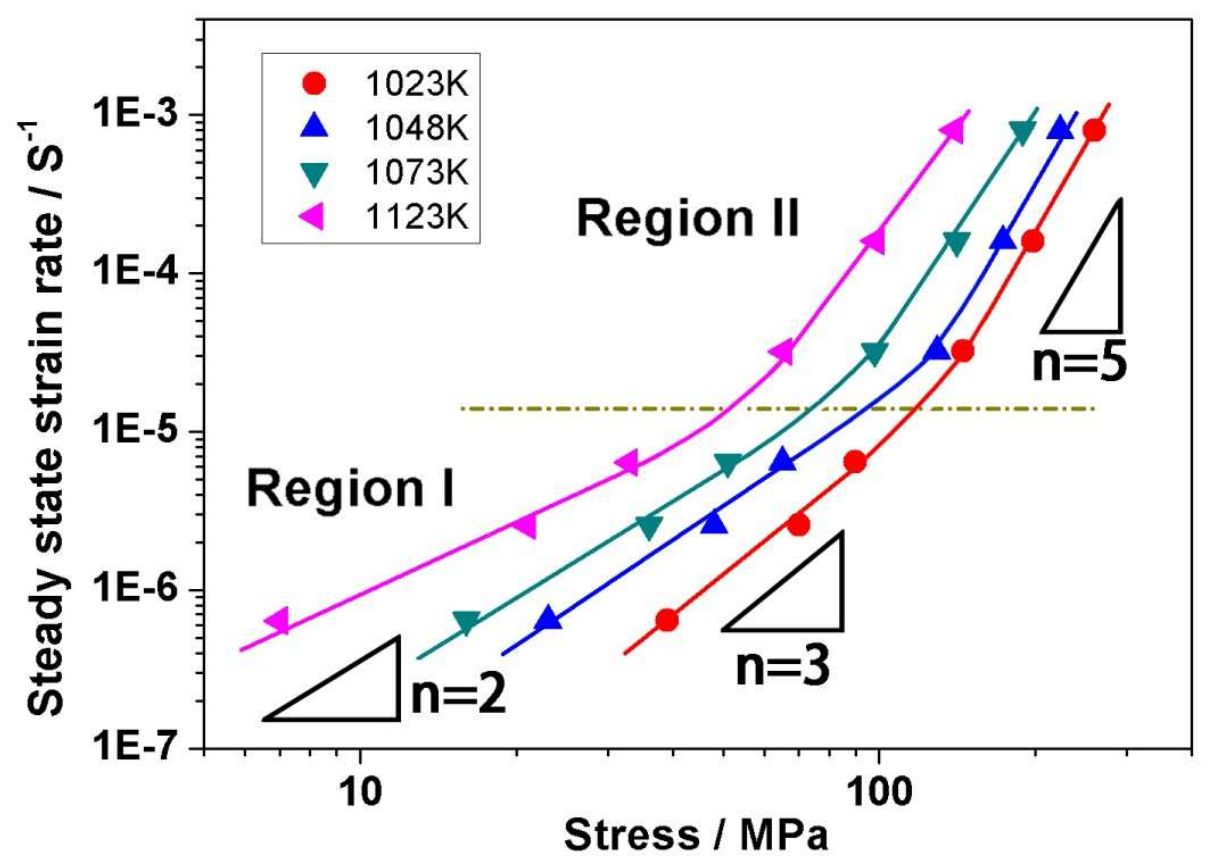

